\title{
Ruyiping formula inhibits metastasis via the microRNA-134-SLUG axis in breast cancer
}

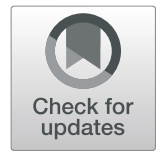

\author{
Ziwei Jiang ${ }^{\dagger}$, Lixia Pei ${ }^{\dagger}$, Ying Xie, Qun Ye, Xiaoqiang Liang, Yiyi Ye and Sheng Liu
}

\begin{abstract}
Background: Metastasis is the leading cause of death among breast cancer patients. MicroRNA-134 has been reported to have a tumor-suppressive role in breast cancer. Ruyiping (RYP), a traditional Chinese formula, has been shown with the ability to reduce breast cancer metastasis in pre-clinical studies. This present study was designed to examine whether miR-134 was involved in RYP-inhibited breast cancer metastasis.

Methods: The expression of SLUG, E-Cadherin, N-Cadherin and miR-134 in MDA-MB-231 and 4T1 cells treated with RYP or vehicle control were determined by quantitative realtime-PCR and western blot. Invasiveness determined by transwell assay as well as SLUG gene expression determined by qPCR were detected in cells transfected with chemically synthesized miR-134 mimics or inhibitors. BALB/C mice were injected with $4 \mathrm{~T} 1$ cells orthotopically and fed with RYP through gavage. Breast tumor growth, metastasis and tumor expression of EMT markers were detected.

Results: Compared with the control, Ruyiping formula significantly inhibited SLUG-regulated breast cancer cells invasion. MiR-134 was induced by RYP in vitro and in vivo and was able to suppress SLUG by targeting its $3^{\prime} U T R$. RYP suppressed SLUG expression and cell invasion through miR-134. In 4T1 tumor-bearing mice, RYP significantly inhibited 4T1 tumor growth and lung metastasis, increased the levels of miR-134 and epithelial marker while decreased the levels of SLUG and mesenchymal marker.
\end{abstract}

Conclusion: Our data uncovered that Ruyiping formula exerts an anti-metastatic activity against breast cancer cells by regulating SLUG through miR-134. MiR-134-SLUG axis might be a promising strategy in breast cancer therapy.

Keywords: Breast cancer, Metastasis, Ruyiping formula, miRNA-134, SLUG, Epithelial-mesenchymal transition

\section{Background}

Breast cancer is a common women malignancy in the world [1] which accounts for 367,900 new diagnosis and 97,972 deaths of Chinese female in 2018 [2]. Although substantial advances in modern therapy such as early diagnosis, chemotherapy and neoadjuvant treatment have improved survival rate of breast cancer, the mortality rate is still high. Metastasis is the major cause leading to treatment failure and patients fatality [3]. Therefore, it is extremely important to understand the molecular

\footnotetext{
* Correspondence: Islhtem1@163.com

${ }^{\dagger}$ Ziwei Jiang and Lixia Pei contributed equally to this work.

Institute of Chinese Traditional Surgery, LongHua Hospital Affiliated to Shanghai University of Traditional Chinese Medicine, 725 Wanpingnan Road, Shanghai 200032, China
}

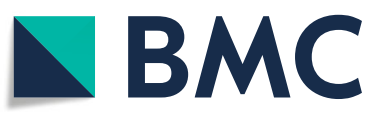

(C) The Author(s). 2021 Open Access This article is licensed under a Creative Commons Attribution 4.0 International License, which permits use, sharing, adaptation, distribution and reproduction in any medium or format, as long as you give appropriate credit to the original author(s) and the source, provide a link to the Creative Commons licence, and indicate if changes were made. The images or other third party material in this article are included in the article's Creative Commons licence, unless indicated otherwise in a credit line to the material. If material is not included in the article's Creative Commons licence and your intended use is not permitted by statutory regulation or exceeds the permitted use, you will need to obtain permission directly from the copyright holder. To view a copy of this licence, visit http://creativecommons.org/licenses/by/4.0/. The Creative Commons Public Domain Dedication waiver (http://creativecommons.org/publicdomain/zero/1.0/) applies to the data made available in this article, unless otherwise stated in a credit line to the data. screen for new, specific and sensitive targets for therapy.

Traditional Chinese herbal medicines have been widely used in clinical practice $[4,5]$. Ruyiping Formula (RYP) is a traditional Chinese medicine (TCM) empirical prescription developed in Longhua Hospital, China. Previous pre-clinical studies have shown that RYP is effective in preventing breast cancer metastasis by protecting the microvascular integrity and suppressing tumor cell growth and migration [6, 7]. It is composed of Iphigenia indica Kunth (Shancigu), Nidus Vespae (Lufengfang), Curcuma phaeocaulis Valeton (Ezhu), raw seeds of Coix lacryma-jobi L. (Shengyiyiren) $12 \mathrm{~g}$ and Akebiae Fructus (Bayuezha). Some components of RYP had been shown with anti-tumor effects, such as Curcumin in Curcuma 
zedoaria and caffeic acid phenethyl ester (CAPE) derived from Nidus Vespae $[8,9]$. However, the specific mechanisms of how RYP inhibits metastasis still remains to be explored.

Metastasis is responsible for more than $90 \%$ of cancerrelated death [10]. EMT plays the major role in cancer metastasis [11]. Multiple cytokines, growth factors and transcription factors have been shown to participate in the EMT progression. SNAI2 (SLUG), a member of zinc-finger transcription factor Snail superfamily, is reported as a marker of malignancy and a key factor in EMT progression [12]. SLUG can be induced by TGF- $\beta$ in cancer cells and then directly binds to the promoter region of E-Cadherin gene to suppress its transcription, thus promotes cancer cell migration $[11,13]$. Therefore, SLUG might be an attractive target for therapeutic modulation of invasiveness in human cancer treatments. However, the molecular mechanism regulating the expression of SLUG is not clearly elucidated.

MicroRNAs are important in translational or posttranscriptional regulation. They are small noncoding RNAs about 20-25 nt in length and bind to the 3 '-untranslated region (UTR) of target mRNAs to exert their inhibition function [14]. Numerous studies have demonstrated that miRNAs participate in tumorigenesis and carcinogenesis. MiR-134, first identified as a brainspecific miRNA [15], was recently reported significantly downregulated in breast cancer cells and reversely correlated with cell proliferation, lymph node metastasis, TNM stages and reduced cell differentiation [16, 17]. Moreover, it was reported to suppress EMT in multiple cancer types $[16,18]$.

The important roles of SLUG and miR-134 in EMT and tumor progression prompt us to hypothesize that they may be involved in RYP-inhibited breast cancer metastasis. In this study, we found that RYP induced miR134 and suppressed SLUG expression in breast cancer both in vitro and in vivo. In the meantime, miR-134 suppressed SLUG 3'UTR activity through its potential binding site. We also demonstrated the importance of miR-134 and SLUG in the RYP-suppressed cancer cell invasion in vitro. Therefore, suppression of SLUG by induction of miR-134 might be the essential mechanism in the RYP-suppressed EMT, a key process involved in RYP-suppressed lung metastasis which was observed in a xenograft model.

\section{Methods}

\section{Materials}

The composition of Ruyiping formula were shown in Table 1 and all ingredients were purchased from Shanghai Kang Qiao Chinese Cut Crude Drug Co.,Ltd. with fixed origin and quality control standards. Morphological, microscopic, and phytochemical identification
Table 1 The composition of Ruyiping formula

\begin{tabular}{lll}
\hline Chinese Name & Latin Name & Origin \\
\hline Ezhu & Curcuma phaeocaulis Valeton & Guangxi \\
Shancigu & Iphigenia indica Kunth & Guizhou \\
Fengfang & Nidus Vespae & Shandong \\
Bayuezha & Akebiae Fructus & Shandong \\
Yiyiren & Coix lacryma-jobi L. & Guizhou \\
\hline
\end{tabular}

were performed by Professor Zhi-Li Zhao from the Department of Pharmacognosy, Shanghai University of Traditional Chinese Medicine. According to the Pharmacopoeia of the People's Republic of China (2015 edition), the rhizome of Curcuma phaeocaulis Valeton, and the mature fruits of Akebiae Fructus were boiled, then air-dried before storage. Pseudobulb of Iphigenia indica Kunth, nest of Vespae Nidus and raw seeds of Coix lacryma-jobi L. were cleansed and air-dried before submitted to storage.. The herbarium voucher specimens were deposited in the Institute of Chinese Traditional Surgery, Longhua Hospital affiliated to Shanghai University of Traditional Chinese Medicine, with voucher numbers SCG-1907033 (Iphigenia indica Kunth), FF-1907034 (Nidus Vespae), EZ-1907035 (Curcuma phaeocaulis Valeton), SYY-1907036 (raw seeds of Coix lacryma-jobi L.) and BYZ-1907037 (Akebiae Fructus).

RPMI-1640 medium and fetal bovine serum were obtained from Gibco (Grand Island, New York, USA). Antibodies against SLUG (A1057), Actin (AC004), ECadherin (A11492), N-Cadherin (A0433) were obtained from ABclonal, Inc. (Wuhan, China). SLUG siRNAs, hsa-microRNA-134 and mmu-microRNA-134 mimics and inhibitors were purchased from Biotend Biotechnologies Co., Ltd. (Shanghai, China).

\section{Preparation of RYP decoction}

RYP decoction was prepared according to previous method [6] with minor modifications. Twelve-gram Iphigenia indica Kunth (Shancigu), $12 \mathrm{~g}$ Nidus Vespae (Lufengfang), 12 g Curcuma phaeocaulis Valeton (Ezhu), $12 \mathrm{~g}$ raw seeds of Coix lacryma-jobi L. (Shengyiyiren) and $9 \mathrm{~g}$ Akebiae Fructus (Bayuezha) were mixed and soaked in $55 \%$ ethanol $(1: 10, \mathrm{w} / \mathrm{v})$ for $4 \mathrm{~h}$ and then refluxed for $1.5 \mathrm{~h}$. After filtering, the decoction was collected and the residues were refluxed again with 55\% ethanol (1:8, w/v) for $1 \mathrm{~h}$. Two parts of decoctions were combined and concentrated, and the concentrated extract was freeze-dried to obtain the RYP extract. The extract was dissolved in dimethyl sulfoxide and diluted with PBS.

\section{Cell culture and stimulation}

Human breast carcinoma cell line MDA-MB-231 and mouse mammary carcinoma cell line $4 \mathrm{~T} 1$ were purchased 
from the Cell Bank of Type Culture Collection of the Chinese Academy of Sciences (Shanghai, China). MDAMB-231 cells were cultured in DMEM medium and 4 T1 cells were cultured in RPMI-1640 medium with $10 \%$ fetal bovine serum at $37^{\circ} \mathrm{C}$ in a $5 \% \mathrm{CO}_{2}$ humidified chamber. Cells were grown to about $70 \%$ confluence before the RYP was used to stimulate cells for $24 \mathrm{~h}$. Cells were collected for RNA isolation or protein extraction.

\section{Animals and experimental pre-metastatic model}

$\mathrm{BALB} / \mathrm{c}$ mice were purchased from SLAC Laboratory Animal Co. Ltd., (Shanghai, China) and raised in individual cages under specific-pathogen-free (SPF) level in the animal facility of Longhua Hospital affiliate to Shanghai University of Traditional Chinese Medicine. Mice were maintained in a temperature-controlled facility with a strict $12 \mathrm{~h}$ light/dark cycles and were given free access to food and water. All animal experiments were approved by the Institutional Animal Care and Use Committee (IACUC) of Longhua Hospital affiliated to Shanghai University of Traditional Chinese Medicine. All the surgeries were performed under anesthesia and all the efforts were made to minimize suffering.

The 4 T1 cells were digested and washed two times by cold PBS. The suspension concentration was adjusted to $2 \times 10^{6}$ cells $/ \mathrm{mL}$. Except for the control group, each mouse was injected $50 \mu \mathrm{L}$ into the fourth mammary fat pads on the right side. RYP extract was gavage administration was given daily for day 8 to day 35 . After anaesthetized with isoflurane, mice were euthanized by cervical dislocation. The tumor growth was photographed and calculated by the formula $V=(W)^{2} \times L / 2$. Metastatic nodules in lung were stained with Bouin's solution. Relative gene expression were detected by realtime PCR or western blot.

\section{SLUG overexpression}

The open reading frames (ORFs) of mouse SLUG were amplified from cDNAs using gene-specific primers. Primers used in present study were shown in Table S1. After subcloning and sequencing, the correct gene fragments were inserted into pCMV-Tag2b. pCMV-Tag2bSLUG plasmids were then transfected into $4 \mathrm{~T} 1$ cells by using Lipofectamine 2000 (Invitrogen). After overnight culture, the transfected cells were treated with RYP. All the information of these DNA sequences was from NCBI database.

\section{Real-time quantitative RT-PCR}

Total RNA was extracted from mouse primary tumors or cells using TRIzol reagent (Life Technologies) following the manufacturer's introduction. Complementary DNA was synthesized using cDNA Reverse Transcriptase kit (Q-111-02, Vazyme). Quantitative real-time (RT)-
PCR-specific primers were designed and validated by NCBI Primer designing tool (https://www.ncbi.nlm.nih. gov/tools/primer-blast/) and all primers are shown in Table S1. Quantitative RT-PCR was performed in triplicate using SYBR green master mix (Vazyme) on a QuantStudio 3 System (Applied Biosystem). The samples with low yield of RNA were pre-determined and excluded. Quantitative analysis was performed using $2^{-\Delta \Delta} \mathrm{Ct}$ method for quantification of the relative mRNA expression.

\section{Immunoblot and immunohistochemistry}

$2 \mathrm{~mm}^{3}$ tumor samples or treated cells were lysed with RIPA buffer ( $\mathrm{pH} 7.4$ ) containing protease inhibitor cocktail (Roche). $20 \mu \mathrm{g}$ of total protein was used for immunoblot. Samples were separated with SDS-PAGE and then transferred to nitrocellulose membranes followed by probing with indicated SLUG, E-Cadherin, N-Cadherin and Actin antibodies. The blots were visualized with ECL Enhanced Kit (Abclonal) on a ChemiDoc Touch Imaging System (Bio-Rad). The densitometry of all the bands was analyzed by Image J and normalized to GAPDH.

For immunostaining, five micrometers of formalinfixed, paraffin-embedded tissue sections were mounted on glass slides. The sections were then dewaxed and subsequent pretreated with antigen retrieval solution. The sections were stained with anti-mouse SLUG antibody (Abclonal) overnight, then incubated with the HRP-labeled goat anti-mouse IgG $(\mathrm{H}+\mathrm{L})$ antibody (1:50 dilution, Beyotime Biotechnology) for 50 min. After DAB staining and hematoxylin counterstaining, sections were dehydrated with graded alcohol, hyalinized in xylene, and finally sealed with neutral gum. Under the microscope, the positive cells were stained brown.

\section{Invasion assay}

24-well transwell plates equipped with the $8 \mu \mathrm{m}$ pore in polyethylene terephthalate membranes (Corning Life Sciences, NY, USA) were used for detection. Matrigel (356,234, Corning Life Sciences) was diluted (1:30) with pre-cooled serum-free medium and added to the precooled filter to form a thin gel layer. After $24 \mathrm{~h}$ treatment of RYP, cells were harvested and diluted to $1 \times 10^{6}$ cells/mL in serum-free medium. $100 \mu \mathrm{L}$ suspension was seeded into the upper chamber. The lower chamber was filled with.

$600 \mu \mathrm{L}$ of medium with $0.5 \%$ FBS. After $24 \mathrm{~h}$ incubation, the cells on the upper surface of the filter were removed by a cotton swab. Cells that invaded to the lower surface were stained with crystal violet and counted by IX71 microscope (Olympus).

\section{Target prediction and luciferase reporter assay}

The SLUG 3'UTR were amplified with specific primers and cloned into pGL3-Control vector with a luciferase 
reporter (Promega). miR-134 binding sites were predicted by TargetScan (http://www.targetscan.org/), miRDB (http://www.mirdb.org/) and miRWalk (http:// mirwalk.umm.uni-heidelberg.de/) database. Reporter plasmid carrying truncations and mutations at the 127 to 133 site (Fig. 3C) were generated by overlap PCR. The primers used were listed in Table S1. To test the activity of SLUG 3'UTR, 4 T1 cells were co-transfected with pGL3-SLUG 3'UTR-Luc plasmids and mmu-miR-134 mimics or inhibitors using Lipofectamine 2000 (Invitrogen). Twenty-four hours post transfection, the cells were collected and luciferase activity was measured on LUMIstar OPTIMA microplate reader (BMG LABTECH) by using the DualLuciferase Reporter Assay system (Promega).

\section{Statistical analysis}

All data are present as mean \pm s.e.m. We did analyses of multiple groups by one-way or two-way ANOVA with Bonferroni post test of GraphPad Prism Version 6. For all statistical tests, we considered $P$ values $<0.05$ to be statistically significant.

\section{Results \\ RYP inhibits invasion through suppressing SLUG expression}

Previous studies showed that RYP inhibits breast cancer lung metastasis. Since EMT play important roles in promoting migration, we thereby examined EMT genes expression in MDA-MB-231 and 4 T1 breast cancer cells after RYP treatment. Compared to the control group, RYP significantly decreased N-Cadherin expression and increased E-Cadherin expression in a dose- and timedependent manner (Fig. 1A-D). Given that SLUG can bind to E-Cadherin promoter to inhibit its transcription, we tested whether RYP can regulate SLUG expression. Interestingly, SLUG was also inhibited in RYP-treated breast cancer cells (Fig. 1A-D). We then confirmed the role of SLUG in RYP-regulated invasion by invasion

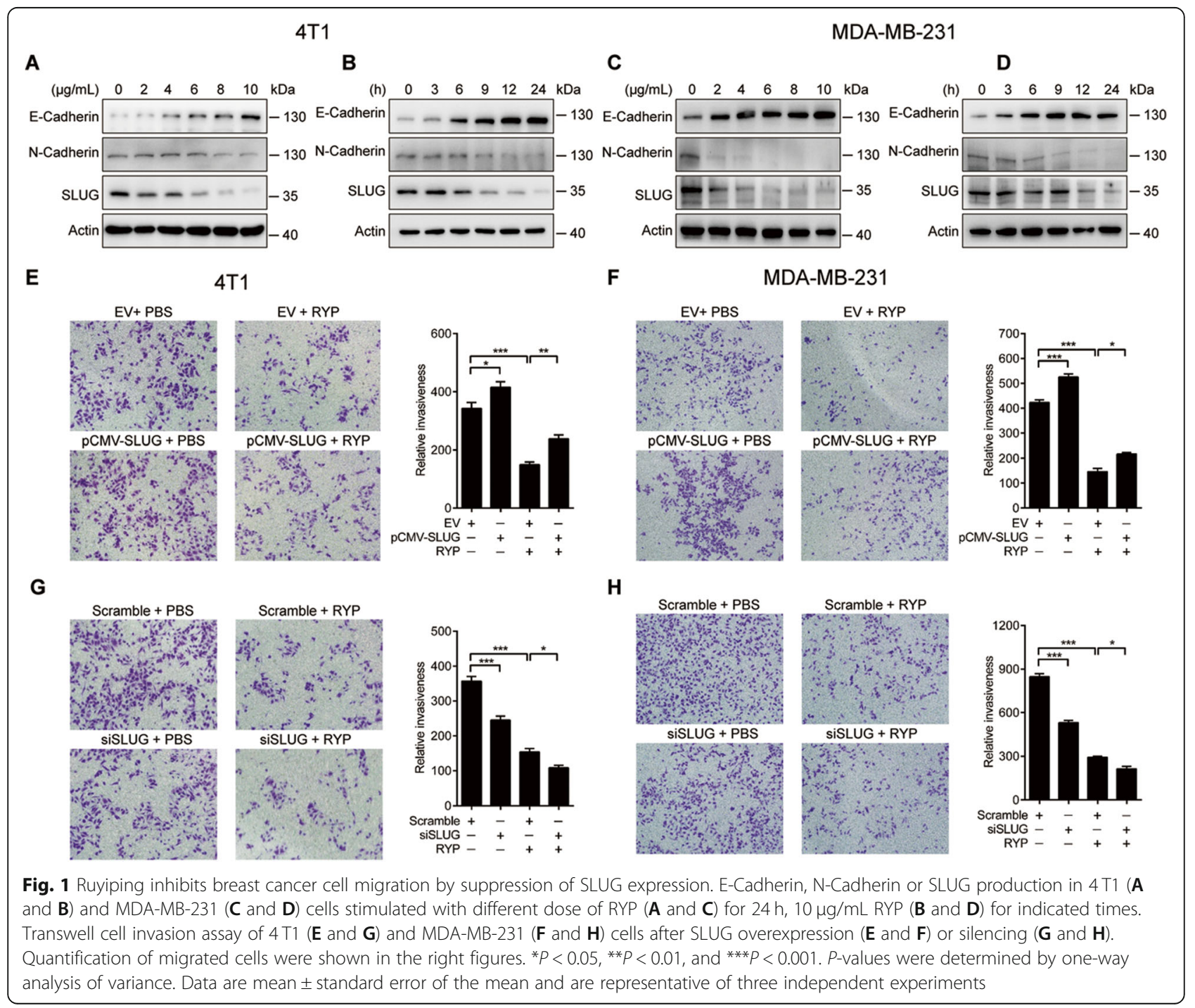


assay using transwell. In both MDA-MB-231 and 4 T1 breast cancer cells, RYP reduced breast cancer cells invasion through the Matrigel. Overexpression of SLUG reversed this RYP-induced suppression of invasion (Fig. 1E-F) while specific SLUG siRNA knockdown enhanced it (Fig. 1G-H). These data demonstrated that RYP inhibits breast cancer cells invasion through suppression of SLUG expression.

\section{RYP induces miR-134 to decrease SLUG translation}

MicroRNAs are important in suppression of gene expression. To further explore the mechanism how RYP inhibits SLUG expression, we used TargetScan (http:// www.targetscan.org/), miRDB (http://www.mirdb.org/) and miRWalk (http://mirwalk.umm.uni-heidelberg.de/) databases to predict possible miRNAs involved in regulating SLUG expression There are 15 miRNAs with high scores in all three databases (Fig. 2A). Eight of them were not reported to regulate SLUG and therefore drew our interest to test whether they are new candidates of SLUG regulator. We further searched their reported relationship with breast cancer and found miR-92a, miR25 and miR-134 to be most studied in breast cancer. Within them, miR-134 has the highest score in all three databases and was our focus in this study.

Therefore we detected pre-miR-134 expression in RYP-treated $4 \mathrm{~T} 1$ cells by qPCR. RYP induces pre-miR134 expression in breast cancer cells in a time- and dose-dependent manner (Fig. 2B and C). To further confirm the role of miR-134, we transfected chemically synthesized miRNA mimics and inhibitors into MDAMB-231 and $4 \mathrm{~T} 1$ cells. SLUG-irrelevant miRNA, miR67 , was used as a negative control. MiR-22 was used as a positive control, which has been reported before as a negative regulator of SLUG. MiR-134 mimics significantly decreased SLUG protein level (Fig. 2D) while miR-134 inhibitors increased SLUG expression (Fig. 2E) which were similar with miR-22 group. Moreover, we used RYP to treat miRNAs-transfected 4 T1 and MDAMB-231 cells. MiR-134 mimics transfection enhanced RYP-induced SLUG reduction (Fig. 2F) while the miR134 inhibitor reversed RYP's function and increased SLUG expression (Fig. 2G). These results demonstrated that RYP inhibits SLUG gene expression through induction of miR-134.

\section{SLUG is a downstream target of miR-134}

We next explored the molecular mechanism by which miR-134 downregulated SLUG expression. 3'UTR region of mRNA is usually where miRNA binds to inhibit the translation. We first co-transfected wild-type SLUG 3'UTR reporter vectors with miR-134 mimics or inhibitors and miR-22 was used as a positive control. Compared to the control group, the reporter luciferase activity with a wild-type SLUG 3'UTR was significantly reduced by miR-22 or miR-134 mimics in $4 \mathrm{~T} 1$ cells (Fig. 3A). However, miR-22 or miR-134 inhibitors enhanced the luciferase activity (Fig. 3B). These results

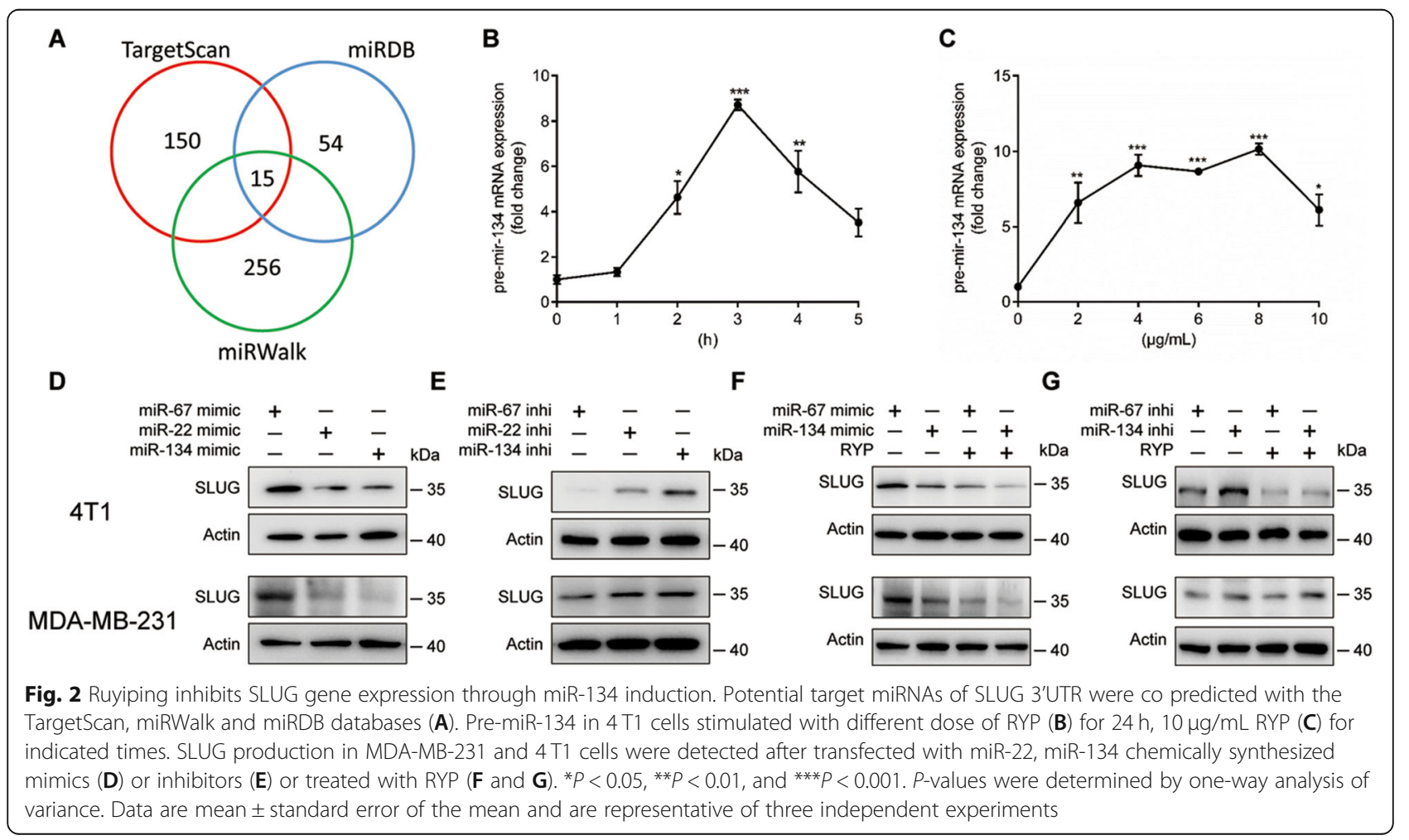



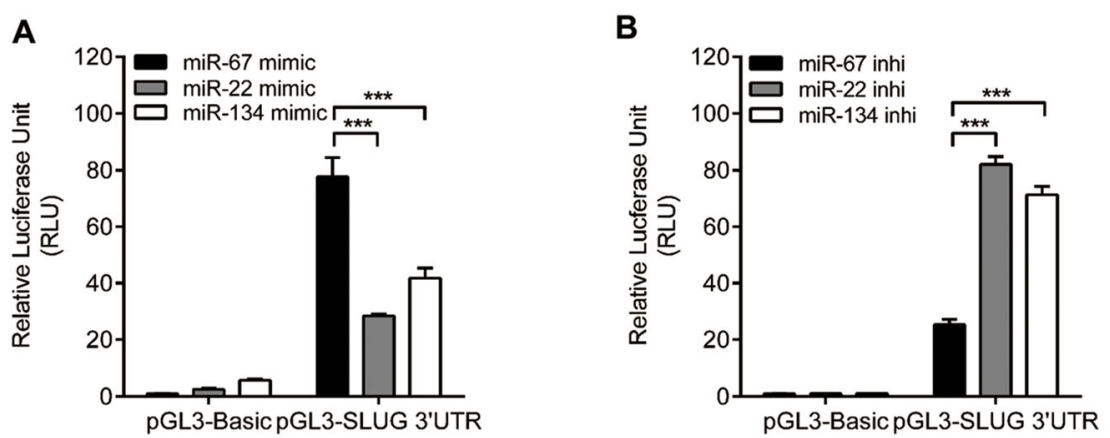

C $\begin{array}{llll}\text { mSLUG 3'UTR WT } & \text { 3' } & 127 \text { CACAAAAGAGTCACACACACAC } & 5, \\ \text { mSLUG 3'UTR Mut } & \text { 3', } & \text { CACAAAAGGACTGTACACACAC } & 5, \\ \text { mSLUG 3'UTR Delta } & \text { 3' } & \text { CACAAAAG_...-ACACACAC } & 5\end{array}$
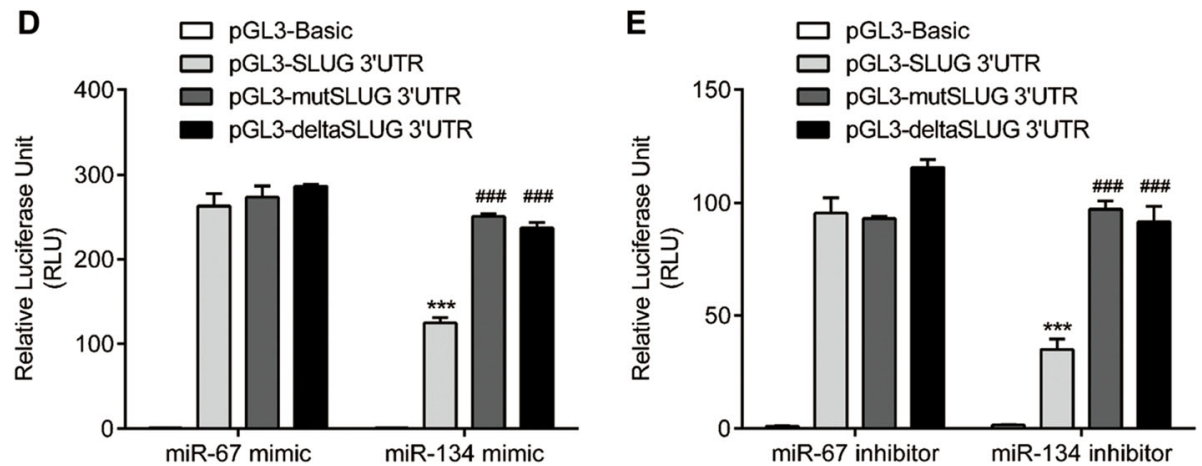

Fig. 3 miR-134 binds to SLUG mRNA 3'UTR to suppress protein translation. Normalized luciferase activity in 4T1 cells transfected with pGL3control-SLUG 3'UTR vector and miR-22, miR-134 chemically synthesized mimics (A) or inhbitors (B). (C) The putative miR-134 binding sites; the wild-type, mutant (Mut) and deletion sequence of 3'-UTR of SLUG. Normalized luciferase activity in 4T1 cells transfected with various reporter vector constructs with miR-67 or miR-134 mimics (D) or inhibitors $(\mathbf{E}){ }^{* * *} P<0.001$, \#\#\#P<0.001. P-values were determined by one-way analysis of variance. Data are mean \pm standard error of the mean and are representative of three independent experiments

confirmed the inhibition of miR-134 in SLUG translation through its 3'UTR. To further confirm the potential binding site of miR-134 in the SLUG 3'UTR, we deleted or mutated it in the SLUG 3'UTR reporter plasmids (Fig. 3C). MiR-134 mimic diminished the reporter's activity with the wild-type SLUG 3'UTR. However, after the potential binding site was deleted or mutated, miR-134 lose its ability to decrease the luciferase activity (Fig. 3D and E). These results suggested that miR-134 downregulates SLUG expression through directly binding to its 3'UTR potential binding site.

RYP inhibits breast cancer cells invasion through miR-134 To further confirm the role of miR-134 in RYP-inhibited breast cancer metastasis, we performed the invasion assay. RYP reduced MDA-MB-231 and 4T1 cells invasion through the Matrigel as demonstrated before. MiR-134 mimics reduced breast cancer cells invasion and enhanced the reduction of invasion by RYP treatment (Fig. 4A and B). In contrast, miR-134 inhibitors increased invasion and aborted RYP's reduction of invasion (Fig. 4C and D). These data demonstrated that miR-134 is an important microRNA involved in RYPinhibited breast cancer cell invasion.

\section{The miR-134/SLUG axis is regulated by RYP in a tumor xenograft model}

To validate the role of miR-134/SLUG axis in RYPregulated breast cancer metastasis, we used a tumor xenograft model by injecting tumor cells into the mammary fat pads of BALB/c female mice. Tumor-bearing mice were detected to have extensive metastatic lesions in the lungs after 35 days, whereas RYP treatment significantly reduced the tumor size (Fig. 5A and B) and suppressed the lung metastasis (Fig. 5C, D and E), indicating that this formula has potential effect of tumor growth suppression. We further detected related gene expression in primary tumors. Consistent with the in vitro experiment results, RYP induced miR-134 and inhibited EMT related SLUG and N-cadherin gene 


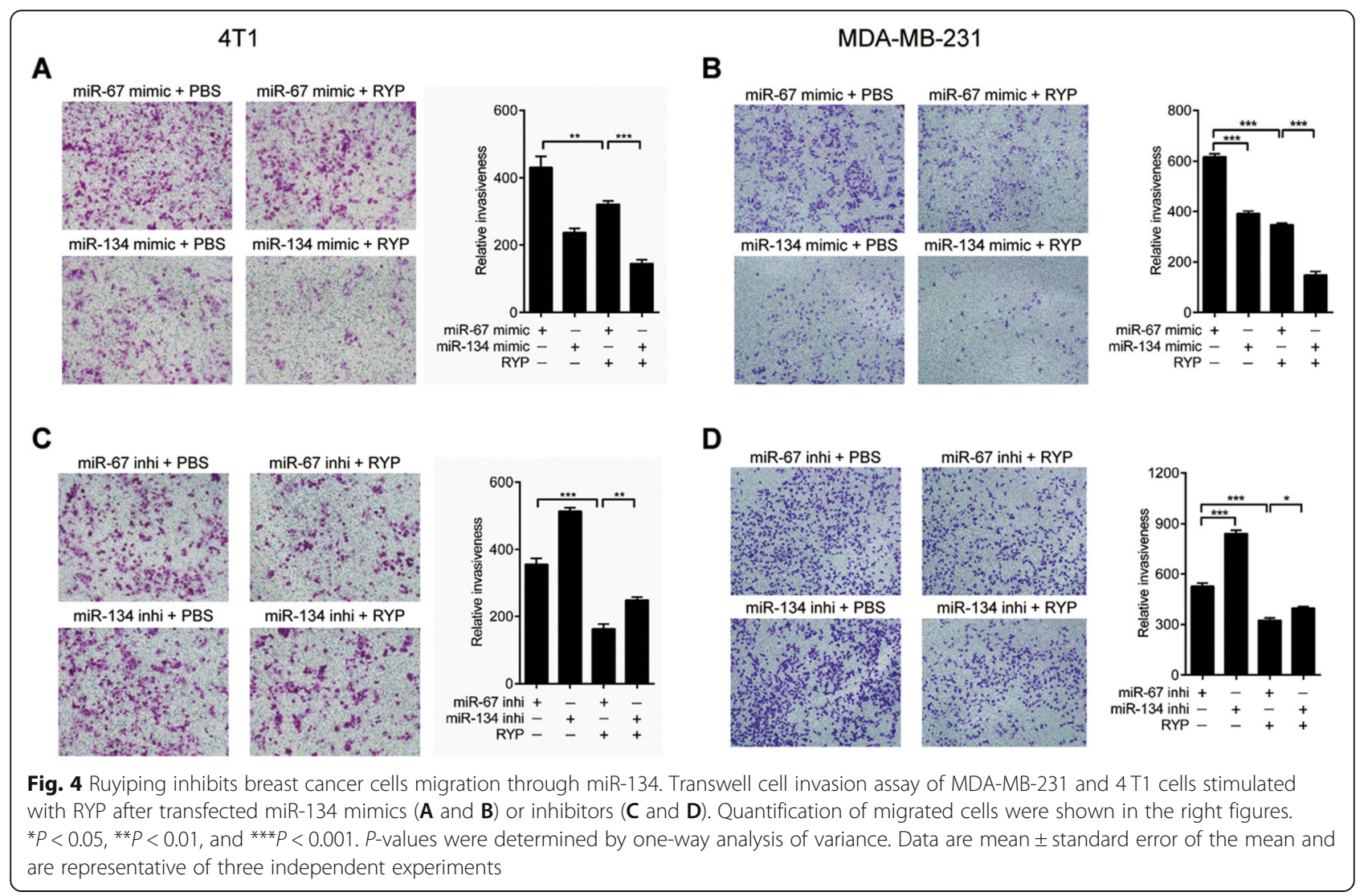

expression while increased E-Cadherin expression (Fig. 5F-K). Altogether, these data supported that RYP induces miR-134 to inhibit SLUG expression and thus suppress EMT, which finally leads to suppression of tumor metastasis.

\section{Discussion}

Chinese herb medicines (CHM) have a long history of clinical use in prevention and treatment of breast cancer [19]. Studies have demonstrated that CHM alleviates side effects and improves life quality of and treatment efficiency. For example, Wenshen Zhuanggu formula mitigates breast cancer bone metastasis through suppression of Jagged1/Notch and TGF- $\beta 1 /$ Smads pathway $[5,20]$. Yiqi formula inhibited the TNBC xenograft tumor growth by reducing p-EGFR and p-AKT1 [21]. Therefore, deeply understanding the mechanisms of $\mathrm{CHM}$ in tumor progression may provide new perspectives for cancer treatment.

Ruyiping formula (RYP) is established by clinical experiences of doctors and has been used in our hospital for more than 30 years with reduced recidivation and metastasis rates in breast cancer patients [22]. Previous pre-clinical studies have shown RYP in protecting the microvascular integrity and suppressing tumor cell growth and migration [6, 7]. Here we revealed a previously unreported function of RYP in regulating breast cancer metastasis via restoring E-Cadherin expression through inhibition of SLUG. The underlying mechanism involves the induction of miR-134, which abrogated SLUG translation by binding with its 3'UTR. Thus, the elucidation of the mechanism of RYP provides crucial information for understanding the traditional Chinese medicine (TCM). Further identification of RYP components and mechanism studies of them in breast cancer metastasis will be an interesting direction for our future studies.

Epithelial-mesenchymal transition (EMT) is a complicated biological process which contributes to tumor progression and metastasis [23]. About 70\% of breast cancer patients with distant metastasis died within five years [24]. Multiple cytokines and growth factors participate in this process [25]. Main characteristics of EMT progression are decreased expression of E-Cadherin and increased expression of N-Cadherin. Epithelial cells lose cell polarity and cell junctions to obtain higher migration, extracellular matrix degrading and invasion abilities [23]. SLUG, a member of zinc finger Snail family, is an important EMT inducer in lung and breast cancer. As a transcriptional repressor, SLUG has been observed to bind to the promoter E-box site together with Lysine Specific Demethylase 1 (LSD1) to repress the expression 


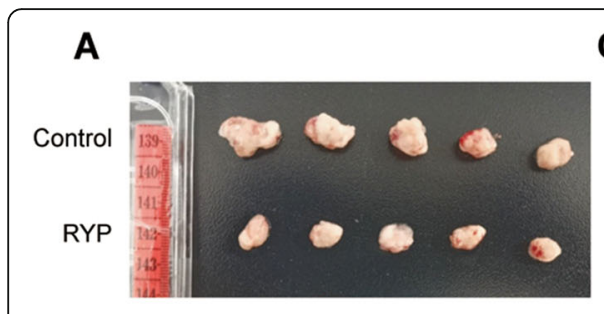

B

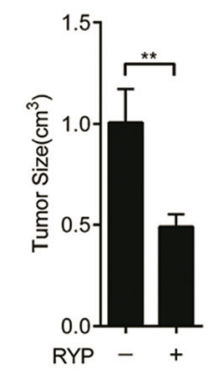

H

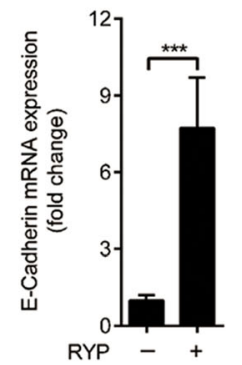

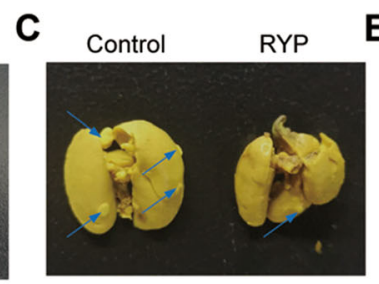

D

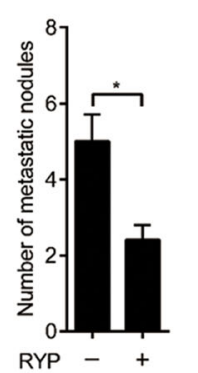

I
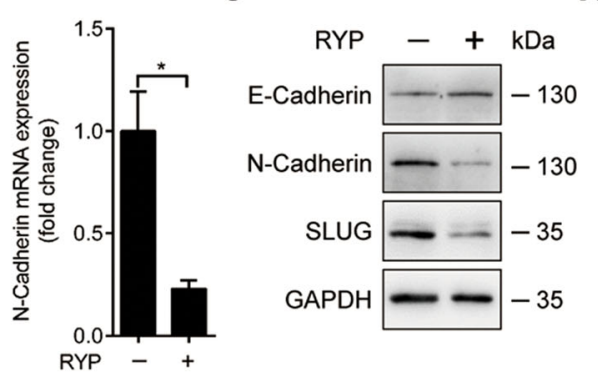

E

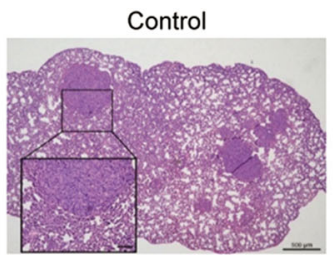

F

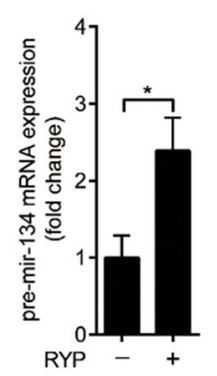

K

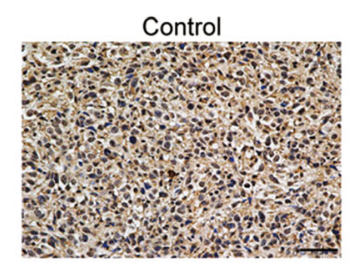

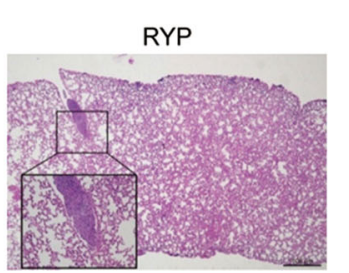

$\mathbf{G}$

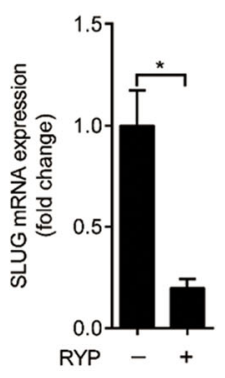

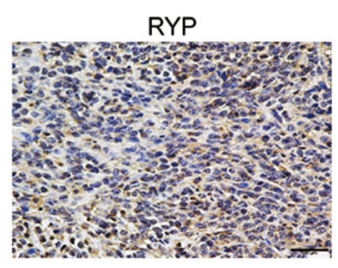

Fig. 5 Ruyiping inhibits breast cancer metastasis in vivo. Mice transplanted with $4 \mathrm{~T} 1$ cells $(n=5)$ and gavage administration once a day with RYP Xenograft tumor growth was photographed and calculated by the formula $V=(W)^{2} \times L / 2(\mathbf{A}$ and $\mathbf{B})$. Metastatic nodules in lung were stained with Bouin's solution and counted (C-E). SLUG, E-Cadherin, N-Cadherin and pre-miR-134 expression in primary tumors were detected by real-time PCR (F-I) or western blot (J). (E) HE staining of lungs five weeks after injection of 4 T1 cells and treated with saline or RYP. Scale bars $=0.5 \mathrm{~mm}$. Arrows designate region of 200x magnification shown in insets. Scale bars $=0.1 \mathrm{~mm}$. (K) Immunohistochemical analysis of SLUG in primary tumors. Scale bars $=0.01 \mathrm{~mm} .{ }^{*} P<0.05,{ }^{* *} P<0.01$. $P$-values were determined by one-way analysis of variance. Data are mean \pm standard error of the mean and are representative of three independent experiments

of E-Cadherin transcription, and thus promote the cancer cells migration [26, 27]. In this study, RYP treatment on breast cancer cells significantly reduced expression of SLUG as well as cell invasiveness compared to the untreated group. SLUG overexpression reversed this effect by RYP, while SLUG-silencing in $4 \mathrm{~T} 1$ and MDA-MB231 cells enhanced the RYP-induced suppression of cell invasiveness. These data were consistent with the previous observations that SLUG-induced EMT [28] and confirmed that RYP inhibits breast cancer cell invasion by reducing SLUG expression.

miRNAs are known to function through gene inhibition and have been reported as significant regulators in diverse human diseases, especially in cancers [29]. In order to identify new SLUG-regulating miRNAs, we used TargetScan, miRDB and miRWalk databases to predict miRNAs binding with SLUG 3'UTR and identified a non-reported SLUG-regulator miR-134, which has high scores in all three databases and has been studied in breast cancer. MiR-134 belongs to chromosome $14 \mathrm{q} 32$ miRNAs clusters and was first reported in dendritic spine development, hippocampal memory and synaptic plasticity $[15,30]$. Recently it is found to be essential for human carcinogenesis, tumor cell proliferation and metastasis and significantly downregulated in breast cancer cells, reversely correlated with cell proliferation, lymph node metastasis, TNM stage and reduced cell differentiation [16, 17]. MiR-134 was also shown to increase breast cancer cells sensitivity to chemotherapy [31] and has been shown to inhibit EMT in multiple cancers, including lung cancer, glioma, breast cancer and colorectal cancer [17]. In our study, we observed that RYP induced miR-134 expression both in vivo and in vitro. The induction of miR-134 peaked at $3 \mathrm{~h}$ of RYP treatment while the suppression of SLUG started to be evident at $6 \mathrm{~h}$, which is consistent with the hypothesis that RYP suppress SLUG through induction of miR-134. Using a 3'UTR luciferase reporter system, we demonstrated 
that miR-134 mimics inhibited the SLUG 3'UTR activity but had no effect when the binding site was mutated or deleted. In contrast, miR-134 inhibitors showed opposite effects. Therefore, we confirmed miR-134 to be a new regulator of SLUG gene through binding to its 3'UTR. Using miR-134 mimics and inhibitors we also confirmed that RYP inhibited SLUG expression and reduced cancer cell invasiveness through induction of miR-134.

In a summary shown in Fig. 6, we proposed the following mechanism by which RYP inhibited the EMT and thus inhibited breast cancer growth and lung metastasis. RYP inhibited SLUG through induction of miR134, which interrupted SLUG translation by binding to its 3'UTR. Inhibition of SLUG resulted in decrease of NCadherin and increase of E-Cadherin, which caused a

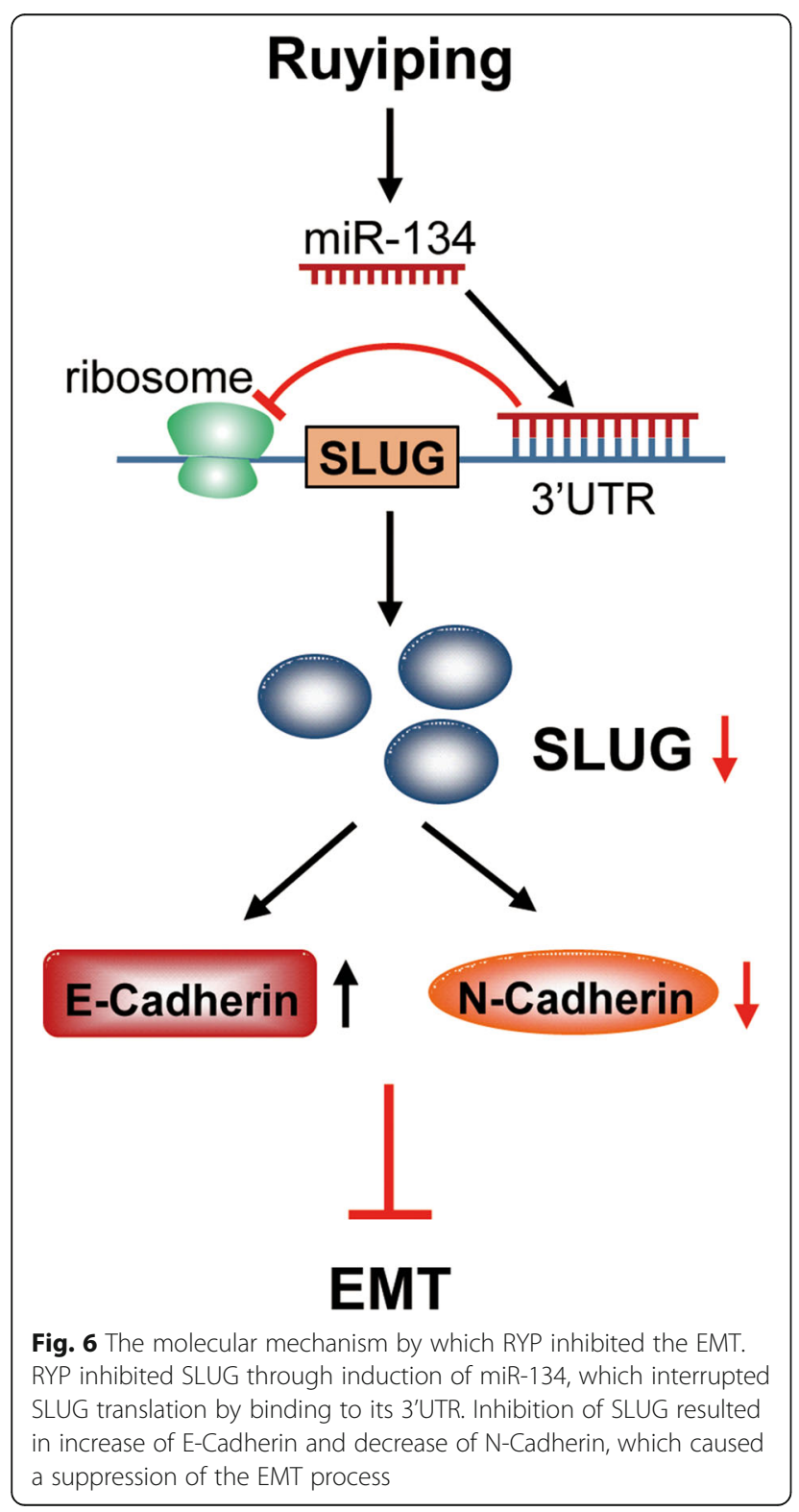

suppression of the EMT process. The in vivo data from mouse model confirmed this mechanism. RYP not only increased miR-134 and decreased SLUG expression in the tumor, but also reduced EMT since E-Cadherin was increased and N-Cadherin was reduced.

Further studies are necessary to explore the tissue distribution and pharmacokinetic mechanism of bioactive compounds from RYP extract in animal models. They will facilitate in predicting various events related to the clinical efficacy and tolerability of this TCM formula against breast cancer metastases.

\section{Conclusions}

In summary, these findings support our discovery that miR-134/SLUG axis is important in RYP-regulated tumor metastasis and the inhibition of the SLUG is due to the interruption of miR-134 at 3'UTR site. MiR-134 might be a potential therapeutic target in metastasis. Moreover, the delineation of the mechanism involved in the inhibition of SLUG expression provides insights into pathways contributing to Ruyiping clinical treatment of breast cancer.

\section{Abbreviations}

RYP: Ruyiping formula; SLUG: snail family transcriptional repressor 2; EMT: Epithelial-mesenchymal transition; 3'UTR: 3'-untranslated region; TCM: traditional Chinese medicine ${ }_{\text {i }}$ CHB: Chinese herb medicines; miR: micro RNA

\section{Supplementary Information}

The online version contains supplementary material available at https://doi. org/10.1186/s12906-021-03365-4.

Additional file 1.

Additional file 2.

\section{Acknowledgements}

Not Applicable.

\section{Authors' contributions}

All authors have made considerable contribution to the work and approved the final version of the submission. $L X P$ and $S L$ conceived and designed the experiments; ZWJ, YX, QY, XQL, YYY performed the experiments; ZWJ and XQL analyzed the data; QY and LXP contributed reagents/materials/analysis tools; ZWJ, YX and SL wrote and reviewed the manuscript.

\section{Funding}

This research was financially supported by Scholars of Longhua Hospital Seedling plan Foundation of Shanghai (LYTD-59), the 2018-2020 Three-year Action Plan for Traditional Chinese Medicine Further Development in Shanghai (ZY (2018-2020)-CCCX-2002-10), National training program for innovative talents of traditional Chinese Medicine.

Availability of data and materials

The datasets used and/or analyzed during the current study are available from the corresponding author on reasonable request. 


\section{Declarations}

\section{Ethics approval and consent to participate}

This study was approved by the Institutional Animal Care and Use Committee (IACUC) of Longhua Hospital affiliated to Shanghai University of Traditional Chinese Medicine.

\section{Consent for publication}

Not Applicable.

\section{Competing interests}

The authors declare that they have no competing financial interests.

Received: 19 October 2020 Accepted: 24 June 2021

Published online: 05 July 2021

\section{References}

1. Woolston C. Breast cancer. Nature. 2015;527(7578):S101. https://doi.org/10.1 038/527S101a.

2. Feng RM, Zong YN, Cao SM, Xu RH. Current cancer situation in China: good or bad news from the 2018 Global Cancer Statistics? Cancer Commun (London, England). 2019:39(1):22.

3. Lorusso $G$, Rüegg C. New insights into the mechanisms of organ-specific breast cancer metastasis. Semin Cancer Biol. 2012;22(3):226-33. https://doi. org/10.1016/j.semcancer.2012.03.007.

4. Han XH, Wang CL, Xie Y, Ma J, Zhang XH, Hu QW, et al. Anti-metastatic effect and mechanisms of Wenshen Zhuanggu formula in human breast cancer cells. J Ethnopharmacol. 2015;162:39-46. https://doi.org/10.1016/j. jep.2014.12.036.

5. Wu C, Chen M, Sun Z, Ye Y, Han X, Qin Y, et al. Wenshen Zhuanggu formula mitigates breast cancer bone metastasis through the signaling crosstalk among the Jagged1/notch, TGF- $\beta$ and IL-6 signaling pathways. J Ethnopharmacol. 2019;232:145-54. https://doi.org/10.1016/j.jep.2018.12.023.

6. Li Z, Sun X, Liu X, Sun Z, Li J. Antitumor effects of Ruyiping on cell growth and metastasis in breast Cancer. Cancer Biother Radiopharm. 2019;34(5): 297-305. https://doi.org/10.1089/cbr.2018.2703.

7. Ye Y, Pei L, Wu C, Liu S. Protective Effect of Traditional Chinese Medicine Formula RP on Lung Microenvironment in Pre-Metastasis Stage of Breast Cancer. Integr Cancer Ther. 2019;18:1534735419876341.

8. Gu X, Zhang Q, Zhang W, Zhu L. Curcumin inhibits liver metastasis of gastric cancer through reducing circulating tumor cells. Aging. 2019;11(5): 1501-9. https://doi.org/10.18632/aging.101848.

9. Wang G, Dong Y, Liu H. Curcumol enhances the anti-tumor effects of metformin via suppressing epithelial-mesenchymal transition in triplenegative breast cancer. Annals of translational medicine. 2020;8(15):946. https://doi.org/10.21037/atm-20-5438.

10. Lambert AW, Pattabiraman DR, Weinberg RA. Emerging biological principles of metastasis. Cell. 2017;168(4):670-91. https://doi.org/10.1016/ j.cell.2016.11.037.

11. Lamouille S, Xu J, Derynck R. Molecular mechanisms of epithelialmesenchymal transition. Nat Rev Mol Cell Biol. 2014;15(3):178-96. https:// doi.org/10.1038/nrm3758.

12. Cobaleda C, Pérez-Caro M, Vicente-Dueñas C, Sánchez-García I. Function of the zinc-finger transcription factor SNAI2 in cancer and development. Annu Rev Genet. 2007:41(1):41-61. https://doi.org/10.1146/annurev.genet.41.1103 06.130146

13. Batlle E, Sancho E, Francí C, Domínguez D, Monfar M, Baulida J, et al. The transcription factor snail is a repressor of E-cadherin gene expression in epithelial tumour cells. Nat Cell Biol. 2000;2(2):84-9. https://doi.org/10.103 $8 / 35000034$

14. He L, Hannon GJ. MicroRNAs: small RNAs with a big role in gene regulation. Nat Rev Genet. 2004;5(7):522-31. https://doi.org/10.1038/nrg1379.

15. Schratt GM, Tuebing F, Nigh EA, Kane CG, Sabatini ME, Kiebler M, et al. A brain-specific microRNA regulates dendritic spine development. Nature. 2006;439(7074):283-9. https://doi.org/10.1038/nature04367.

16. Su X, Zhang L, Li H, Cheng P, Zhu Y, Liu Z, et al. MicroRNA-134 targets KRAS to suppress breast cancer cell proliferation, migration and invasion. Oncol Lett. 2017:13(3):1932-8. https://doi.org/10.3892/ol.2017.5644.

17. Pan JY, Zhang F, Sun CC, Li SJ, Li G, Gong FY, et al. miR-134: a human Cancer suppressor? Mol Ther Nucleic Acids. 2017;6:140-9. https://doi.org/1 0.1016/j.omtn.2016.11.003.
18. Peng SY, Tu HF, Yang CC, Wu CH, Liu CJ. miR-134 targets PDCD7 to reduce E-cadherin expression and enhance oral cancer progression. Int J Cancer. 2018;143(11):2892-904

19. Kai W, Yating S, Lin M, Kaiyong Y, Baojin H, Wu Y, et al. Natural product toosendanin reverses the resistance of human breast cancer cells to adriamycin as a novel PI3K inhibitor. Biochem Pharmacol. 2018;152:153-64. https://doi.org/10.1016/j.bcp.2018.03.022.

20. Ma J, Li J, Wang Y, Chen W, Zheng P, Chen Y, et al. WSZG inhibits BMSCinduced EMT and bone metastasis in breast cancer by regulating TGF- $\beta 1 /$ Smads signaling. Biomed Pharmacother. 2020;121:109617.

21. Liao MJ, Ye MN, Zhou RJ, Sheng JY, Chen HF. Yiqi formula enhances the antitumor effects of erlotinib for treatment of triple-negative breast cancer xenografts. eCAM. 2014;2014:628712.

22. Liu S, Hua YQ, Sun ZP, Tan S, Lu DM: [clinical observation of Ruyiping in preventing recidivation and metastasis of breast cancer]. Zhong xi yi jie he xue bao = J Chin Integr Med 2007, 5(2):147-149, DOl: https://doi.org/10.373 6/jcim20070209.

23. Shook D, Keller R. Mechanisms, mechanics and function of epithelialmesenchymal transitions in early development. Mech Dev. 2003;120(11): 1351-83. https://doi.org/10.1016/j.mod.2003.06.005.

24. Steeg PS. Targeting metastasis. Nat Rev Cancer. 2016;16(4):201-18. https:// doi.org/10.1038/nrc.2016.25.

25. Eckhardt BL, Francis PA, Parker BS, Anderson RL. Strategies for the discovery and development of therapies for metastatic breast cancer. Nat Rev Drug Discov. 2012;11(6):479-97. https://doi.org/10.1038/nrd2372.

26. Phillips S, Kuperwasser C. SLUG: critical regulator of epithelial cell identity in breast development and cancer. Cell Adhes Migr. 2014;8(6):578-87. https:// doi.org/10.4161/19336918.2014.972740.

27. Herranz N, Pasini D, Díaz VM, Francí C, Gutierrez A, Dave N, et al. Polycomb complex 2 is required for E-cadherin repression by the Snail1 transcription factor. Mol Cell Biol. 2008;28(15):4772-81. https://doi.org/10.1128/MCB. 00323-08

28. Shao S, Zhao X, Zhang X, Luo M, Zuo X, Huang S, et al. Notch1 signaling regulates the epithelial-mesenchymal transition and invasion of breast cancer in a Slug-dependent manner. Mol Cancer. 2015;14(1):28. https://doi. org/10.1186/s12943-015-0295-3.

29. Wong CM, Tsang FH, Ng IO. Non-coding RNAs in hepatocellular carcinoma: molecular functions and pathological implications. Nat Rev Gastroenterol Hepatol. 2018;15(3):137-51. https://doi.org/10.1038/nrgastro.2017.169.

30. Chen T, Yang YJ, Li YK, Liu J, Wu PF, Wang F, et al. Chronic administration tetrahydroxystilbene glucoside promotes hippocampal memory and synaptic plasticity and activates ERKs, CaMKII and SIRT1/miR-134 in vivo. J Ethnopharmacol. 2016;190:74-82. https://doi.org/10.1016/j.jep.2016.06.012

31. O'Brien K, Lowry MC, Corcoran C, Martinez VG, Daly M, Rani S, et al. miR-134 in extracellular vesicles reduces triple-negative breast cancer aggression and increases drug sensitivity. Oncotarget. 2015;6(32):32774-89. https://doi.org/1 0.18632/oncotarget.5192.

\section{Publisher's Note}

Springer Nature remains neutral with regard to jurisdictional claims in published maps and institutional affiliations.

Ready to submit your research? Choose BMC and benefit from:

- fast, convenient online submission

- thorough peer review by experienced researchers in your field

- rapid publication on acceptance

- support for research data, including large and complex data types

- gold Open Access which fosters wider collaboration and increased citations

- maximum visibility for your research: over $100 \mathrm{M}$ website views per year

At BMC, research is always in progress.

Learn more biomedcentral.com/submission 